\title{
Development of a Sampling Collection Device with Gynecology-based Diagnostic
}

Procedures

Jhih-Yan Cheng ${ }^{1}{ }_{3^{*}}$ Mow-Jung Feng ${ }^{2}$, Chia-Chi Wu ${ }^{2}$, Jane Wang ${ }^{1}$, Ting-Chang Chang ${ }^{2 *}$, Chao-Min Cheng ${ }^{3}$

${ }^{1}$ Department of Chemical Engineering, National Tsing Hua University, Hsinchu 300, Taiwan

${ }^{2}$ Department of Obstetrics and Gynecology, Chang Gung Memorial Hospital Linkou Branch, Taoyuan 333, Taiwan

3

Institute of Biomedical Engineering, National Tsing Hua University, Hsinchu 300, Taiwan

Corresponding author: Chao-Min Cheng, Institute of Biomedical Engineering, National Tsing Hua University, Hsinchu 300, Taiwan, e-mail: chaomin@mx.nthu.edu.tw

Ting-Chang Chang, Department of Obstetrics and Gynecology, Chang Gung Memorial Hospital Linkou Branch, Taoyuan 333, Taiwan, e-mail: tingchang.chang@gmail.com

\section{ABSTRACT}

Cervicovaginal fluid plays an important role in the detection of many female genital diseases, but the lack of suitable collection devices in the market severely challenges test success rate. Appropriate clinical sampling devices for cervicovaginal fluid collection would help physicians detect diseases and disease states more rapidly efficiently, and accurately. The objective of this study was to develop a readily usable sampling collection device that would eliminate macromolecular interference and accurately provide specimens for further studies. This study was designed to develop an effective device to collect cervicovaginal fluid from women with symptoms of endometrial lesions, women appearing in the clinic for a routine Papanicolaou smear, and/or women seeking a routine gynecologic checkup. Paper-based assay, ELISA, and qNano were used to provide accurate diagnoses. A total of 103 patients successfully used the developed device to collect cervicovaginal fluid. Some of the collected specimens were used to detect glycogen, lactate, and $\mathrm{pH}$ for determining pathogen infection. Other specimen samples were tested for the presence of female genital cancer by comparing interleukin 6 concentration and microvesicle concentration. We proposed a noninvasive screening test for the diagnosis of female genital diseases using a dualmaterial collection device. The outer, non-woven fabric portion of this device was designed to filter macromolecules, and the inner cotton portion was designed to absorb cervicovaginal fluid. 


\section{Supporting Information}

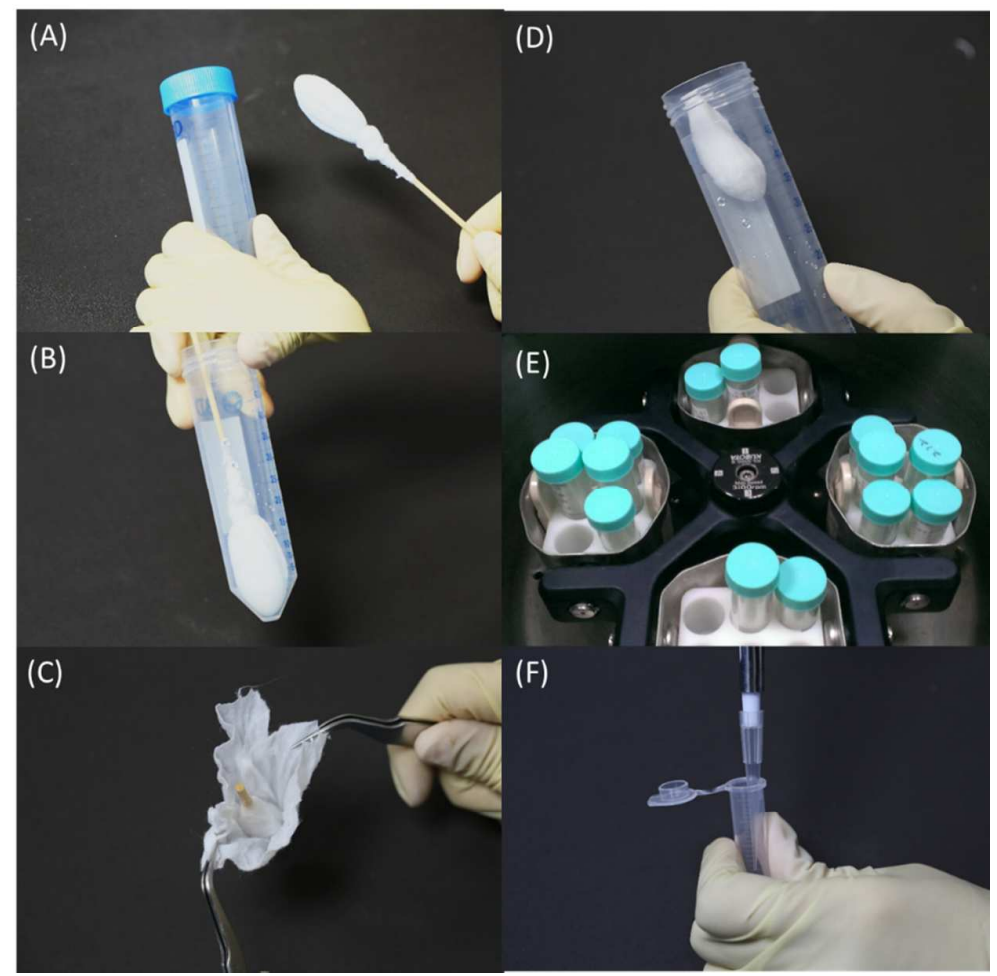

Figure 1.Sample recovery procedure (a)\&(b) Before collecting cervicovaginal fluid, the device was soaked in in sodium chloride $(\mathrm{NaCl})$ solution. (c) The outer filter was discard after collecting cervicovaginal fluid. (d)\&(e) The cotton swab was placed in the tube and centrifuged (5000 g, $10 \mathrm{~min}$ ) to collect the specimen. (f) The liquid was kept as the specimen for further diagnosis or storage. 


\section{Lactate assay standard curve}

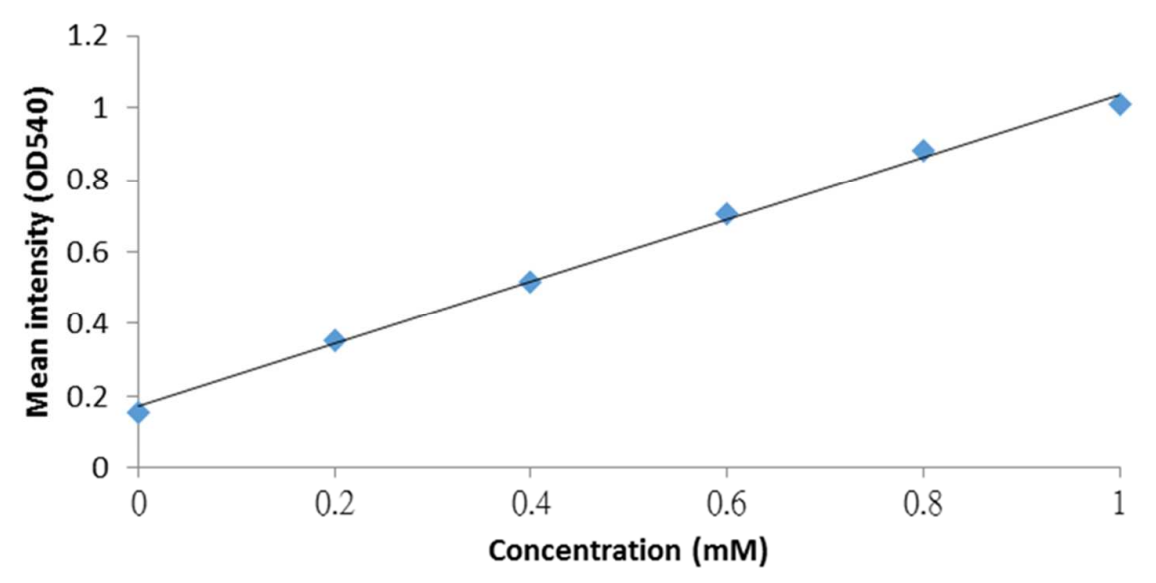

Figure 2.Analytical calibration curve for the lactate in standard solution. $\left(\mathrm{R}^{2}=0.9969\right)$ The detection of lactate concentration range from $0-1 \mathrm{mM}$. 
(a)

Glycogen standard curve

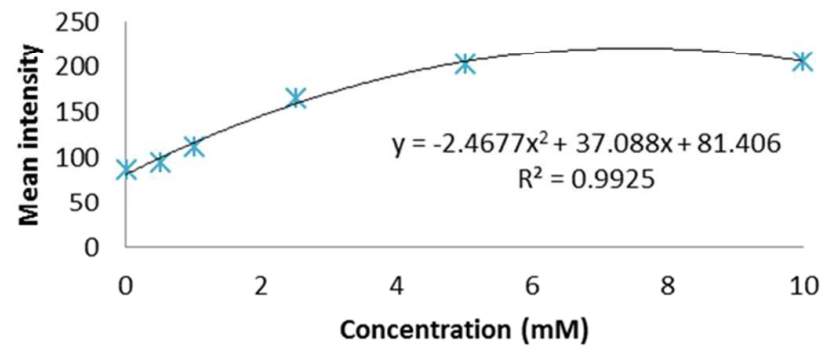

(b)

\section{Lactate standard curve}

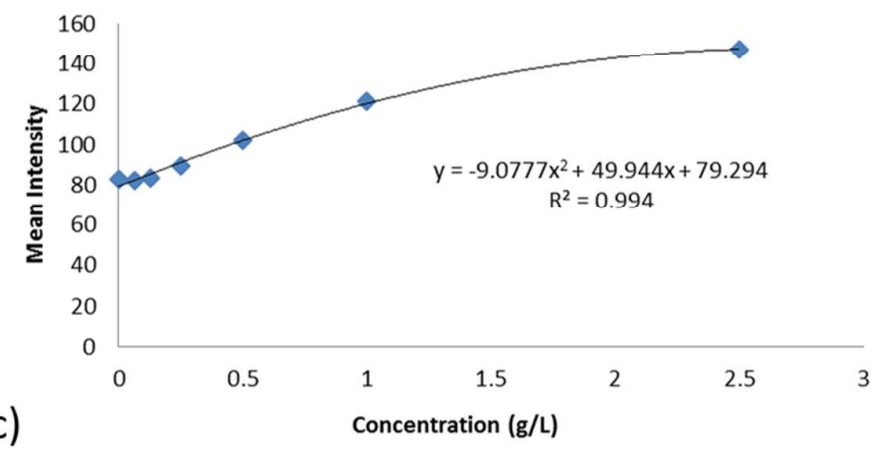

\begin{tabular}{|c|c|c|c|c|c|c|}
\hline $\mathrm{pH}$ value & 3 & 4 & 5 & 6 & 7 & 8 \\
\hline color & 0 & 0 & 0 & 0 & 0 & 0 \\
\hline
\end{tabular}

Figure 3. (a) Analytical calibration curve for the glycogen in standard solution. $\left(\mathrm{R}^{2}=0.9925\right)$ The detection of glycogen concentration range from 0-10mM. (b) Analytical calibration curve for the lactate in standard solution $\left(\mathrm{R}^{2}=0.994\right)$ The detection of lactate concentration range from $0-2.5 \mathrm{~g} / \mathrm{L}$. (c) The color change at different $\mathrm{pH}$ value $(\mathrm{pH}=3-8)$ 


\section{IL-6 assay standard curve}

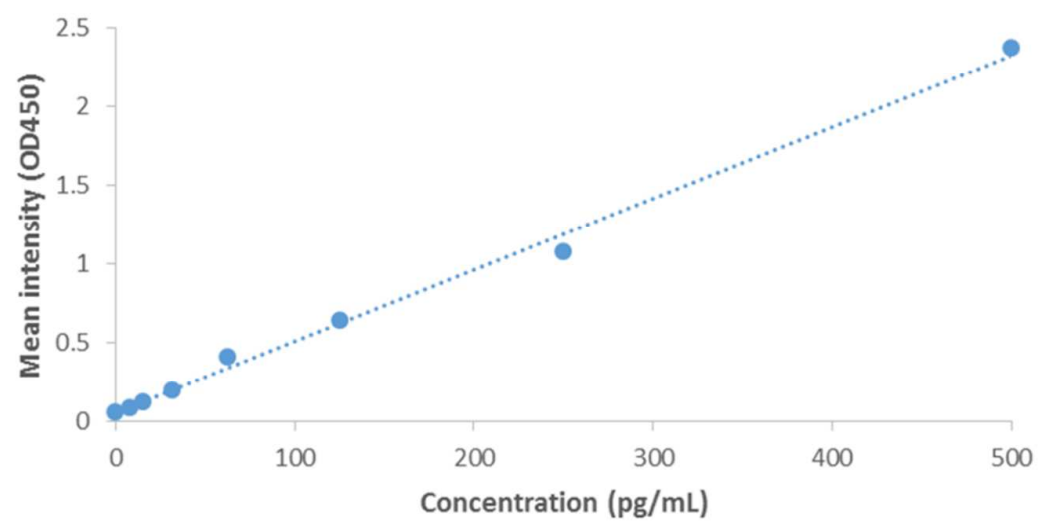

Figure 4.Analytical calibration curve for the IL-6 in standard solution measured using ELISA kit. $\left(\mathrm{R}^{2}=0.9951\right)$ The detection of IL-6 concentration range from $0-500 \mathrm{pg} / \mathrm{mL}$. 